\title{
Evaluation of cerebrospinal fluid flow dynamic changes in patients with idiopathic intracranial hypertension using phase contrast cine MR imaging
}

\author{
Tamer Belal $^{1^{*}}$ (D), Abd-Elhalim Al Tantawy ${ }^{1}$, Fatema Mohamed Sherif ${ }^{2}$ and Alshaimaa Ramadan ${ }^{1}$
}

\begin{abstract}
Background: Idiopathic intracranial hypertension $(\mathrm{IIH})$ mainly affects overweight women in the middle age period. The pathophysiology of IIH stays unclear, but suggested mechanisms include excess CSF production, reduced CSF absorption, increased brain water content, and increased cerebral venous pressure

Objectives: To assess the cerebrospinal fluid (CSF) flow dynamic changes in aqueduct of Sylvius in patients of idiopathic intracranial hypertension (IIH) with new MRI technique: phase contrast cine MRI (PCC-MRI).

Methods: Thirty patients diagnosed with idiopathic intracranial hypertension were divided into 3 groups according to treatment options (no treatment, medical treatment, and medical treatment with repeated lumbar tapping). CSF flow data were evaluated by phase contrast cine MRI.

Results: PCC-MRI parameters were significantly higher in group who was on medical treatment (group II) than other groups. The sensitivity of PCC MRI parameters ranged from 56.7 (stroke volume (SV) and mean flow (MF)) to 83.3\% (peak systolic velocity (PSV)). A statistically significant difference was found for the mean flow value ( $p$ 0.039) between the control group and IIH patients.

Conclusion: The most specific CSF flowmetry parameter detected to help diagnosis of $\mathrm{IH}$ is mean flow especially among early discovered patients. PCC MRI can be used as non-invasive technique for diagnosis of $\mathrm{IH}$ and treatment follow-up.
\end{abstract}

Keywords: Cerebrospinal fluid, Idiopathic intracranial hypertension, Phase contrast cine magnetic resonance imaging

\section{Introduction}

Idiopathic intracranial hypertension (IIH) is a disease with no clear etiology that mainly affects overweight women in the middle age period with incidence of 1-2/ 100,000 [1]. Headache, visual problems, bilateral papilledema, and pulsatile tinnitus are the most common $\mathrm{IIH}$ symptoms, and permanent visual loss is the most

\footnotetext{
* Correspondence: tamr200@yahoo.com

'Department of Neurology, Faculty of Medicine, Mansoura University, Mansoura 35516, Egypt

Full list of author information is available at the end of the article
}

important complication [2]. The pathophysiology of IIH stays unclear, but suggested mechanisms include excess cerebrospinal fluid (CSF) production, reduced CSF absorption, increased brain water content, and increased cerebral venous pressure [3].

The radiological findings of IIH include empty sella, perioptic subarachnoid space enlargement, and flattening of the posterior aspect of the globe [4].

The research of CSF flow has gained an attention especially in the recent years by using phase contrast cine magnetic resonance imaging (PCC-MRI); which is very

\section{Springer Open}

(c) The Author(s). 2020 Open Access This article is licensed under a Creative Commons Attribution 4.0 International License, which permits use, sharing, adaptation, distribution and reproduction in any medium or format, as long as you give appropriate credit to the original author(s) and the source, provide a link to the Creative Commons licence, and indicate if changes were made. The images or other third party material in this article are included in the article's Creative Commons licence, unless indicated otherwise in a credit line to the material. If material is not included in the article's Creative Commons licence and your intended use is not permitted by statutory regulation or exceeds the permitted use, you will need to obtain permission directly from the copyright holder. To view a copy of this licence, visit http://creativecommons.org/licenses/by/4.0/. 
sensitive even for very slow flows. PCC-MRI makes it possible to show the CSF flow depending on anatomical structures and phase sensitive methods and allows for quantitative assessment of the flow. Related studies have focused on structures such as aqueduct of Sylvius, where the flow is the most regular, as well as the ventricular system and subarachnoid spaces $[5,6]$.

It is important to make a good assessment whether the abnormalities seen using PCC-MRI for changes in CSF flow dynamic parameters were due to the disease itself or secondary to medications or repeated spinal tapping [6].

\section{Aim of the work}

To evaluate CSF flow dynamic changes in aqueduct of Sylvius among patients with idiopathic intracranial hypertension (IIH) using novel MRI tool, phase contrast cine MRI (PCC-MRI), and how it can help as a diagnostic tool.

\section{Methods}

A case control study was conducted on 30 patients confirmed to have IIH according to the modified Dandy criteria [7] at the Neurology Department. These patients were either first discovered to have IIH or in a regular follow-up at our outpatients' clinic. All are females with age range from 18 to 42 years. Ten patients were already on medical treatment only (group II), ten cases were on medical treatment and frequent lumbar tapping (group III) with regular follow-up at our outpatient clinic for 2 consecutive years, and other ten cases were diagnosed with $\mathrm{IIH}$ recently but had not yet started any medications (group I). Ten healthy female subjects were subjected to our study as a control with age range from 23 to 41 years (group IV). All patients underwent detailed history, full neurological examination, fundus examination, measurement of CSF opening pressure by lumbar puncture, neuro-imaging, and other laboratory findings. The study received approval from the Institutional Review Board, Faculty of Medicine, with code number MS/17.07.13. Informed consents were obtained from all participants in the study.

We recruited patients with IIH according to diagnostic criteria for pseudotumor cerebri syndrome [8]: (1) papilloedema, (2) normal neurologic examination except for cranial nerve abnormalities, (3) neuroimaging: normal brain parenchyma without evidence of hydrocephalus, mass, or structural lesion and no abnormal meningeal enhancement and magnetic resonance venography for others, (4) normal CSF composition, (5) elevated lumbar puncture opening pressure $\left(>250 \mathrm{~mm} \mathrm{H}_{2} \mathrm{O}\right.$ ).

Those patients who had abnormal magnetic resonance imaging (MRI) findings of space-occupying lesions, hydrocephalus, meningitis, or subarachnoid hemorrhage were excluded from this study. Also, those who had normal CSF pressure or had disc edema from other causes as ischemic optic neuropathy and diabetic papillopathy were excluded from the study. Finally, patients who had contraindication for MRI were excluded from the study such as: presence of cardiac pacemaker or those who had electrically or magnetically activated implants (cochlear implants).

The study was conducted on a 1.5-T MRI scanner (Ingenia, Philips Healthcare 1.5 T S E) using a circular polarized head coil. CSF flow dynamic quantitative study was performed using high-resolution axial phase-contrast protocol with imaging phase perpendicular to cerebral aqueduct. A cine acquisition with sensitivity to velocity in the section-select direction was obtained. The direction of flow encoding was caudocranial. The acquired raw data were interpolated to produce 16 frames equally spaced in the cardiac cycle by means of an MRI-compatible peripheral pulse transducer applied to the subject's finger with retrospective cardiac gating that means MR data are acquired continuously (not in response to a particular cardiorespiratory "trigger" event).

After the data acquisition, all images were transferred to the workstation equipment with the Q flow software that permits quantification of flow-related physiologic data like CSF. A region of interest was drawn manually to include all pixels that reflected the CSF flow signals of the aqueduct on the phase images after magnification of the images to clarify the aqueduct, after that the CSF flow parameters were automatically extracted.

Firstly, the images were analyzed qualitatively using midsagittal phase and magnitude images which revealed aqueductal CSF flow in both systole and diastole; caudal flow in systole was represented as hyperintense signal, while the diastolic flow (cranial flow) was represented as hypointense signal; the change of flow in the aqueduct during systole and diastole ruled out the presence of aqueductal obstruction [9].

For quantitative assessment, peak systolic and diastolic velocities were extracted from the mean velocity time curve. CSF flow dynamics were analyzed in terms of peak diastolic velocity (PDV) in centimeter per second, peak systolic velocity (PSV) in centimeter per second, and aqueductal area. Aqueductal stroke volume was calculated by summation of forward and backward flow, multiplied by duration of CSF flow. The mean flow (MF) was calculated by multiplying the mean velocity in centimeter per second by the aqueductal area in square centimeters where the mean velocity was automatically determined from the mean value of the measured velocities of each cardiac cycle [10]. 


\section{Statistical analysis}

Data were fed to the computer and analyzed using IBM Statistical Package for Social Sciences (SPSS) software package version 22.0 [11]. Qualitative data were described as numbers and percentages using Chi-square test and Monte Carlo test. Quantitative data were described using mean \pm standard deviation (SD) for parametric data using Student's $t$ test and one way analysis of variance (ANOVA) test. Significance of the obtained results was judged at the (0.05) level. The accuracy of a test to discriminate diseased cases from non-diseased cases is evaluated using receiver operating characteristic (ROC) curve analysis. Sensitivity and specificity were detected from the curve; positive predictive value (PPV), negative predictive value (NPV), and accuracy were calculated through cross tabulation.

\section{Results}

Patients were classified in this study into 4 groups: group I, first discovered (naïve) IIH patients (10 patients), group II, known IIH patients on medical treatment (10 patients), group III, known IIH patients on medical treatment and frequent lumbar tapping (10 patients). Ten healthy controls matched with the age and sex were included and considered as group IV. All patients were females with overall age ranged from 18 to 42 years. There were no significant group differences in age $(p$ value $=0.41)$ and body mass index $(p$ value $=0.09)$ (Table 1).

PCC-MRI parameters including mean aqueductal area, peak systolic velocity (PSV), peak diastolic velocity (PDV), the mean flow (MF), and the stroke volume (SV) were assessed.

There was statistically significant difference between group II (on medical treatment) and the control group regarding the PDV and between all studied groups regarding the mean flow. The MF was significantly higher in group I than other groups. The PSV, PDV, and SV were significantly higher in group who was on medical treatment (group II) than other groups (Table 2).

For measuring the sensitivity and specificity of each PCC-MRI parameters and opening CSF pressure using ROC curve, opening pressure had the highest sensitivity (92.6\%) and specificity (100\%) with accuracy reaching 94.6\% when the AUC (area under the curve) was excellent $(=1)$ and cutoff point for it was $\geq 26 \mathrm{~cm} \mathrm{H}_{2} \mathrm{O}$. The AUC values for other PCC-MRI parameters (PSV, PDV,
MF, SV, and aqueduct area) were poor with lower sensitivity and specificity values and lower accuracy as shown in Table 3.

After adjusting confounding factors using logistic regression analysis, we found that SV and aqueductal area are independent predictors of MF among studied cases Table 4.

\section{Discussion}

PCC-MRI CSF flow study has gained interest over the last 2 decades. Previous studies used PCC-MRI for assessment of CSF dynamic in different disorders other than IIH, especially normal pressure hydrocephalus $(\mathrm{NPH})$, Arnold-Chiari malformations, and sometimes multiple sclerosis [12]. According to our knowledge, very few studies were concerned with the assessment of CSF flow dynamics in patients with IIH [13].

Akay and his colleagues compared the PCC-MRI parameters of the patients recently diagnosed with IIH and those who were untreated with those improved on medical treatment protocols using the following parameters (aqueductal area, mean rate, peak rate, mean flow) [12]. If the mean rate and flow was used as a comparison between patients with IIH and controls, significant difference was noted. This difference was secondary to a striking reduction in the mean rate and flow values in the patients who improved after medical treatment in comparison to both the healthy subjects and the newly diagnosed patients. Also, the same differences were noted in untreated group as higher rates were noted.

In our study, there was statistically significant difference between the group on medical treatment and the control group regarding the PDV and between all studied groups regarding the mean flow. The MF was significantly higher in the 1st discovered group than other groups. The PSV, PDV, and SV were significantly higher in the group who was on medical treatment than other groups. This difference was attributed to a marked reduction in the mean rate and flow values in the patient group with improved symptoms after treatment compared to both the control group and the recently diagnosed untreated group.

PSV has higher sensitivity $(=83.3 \%)$ than other CSF flowmetry parameters with accuracy $(=77.5 \%)$. On the other hand, MF has higher specificity $(=80 \%)$ than other CSF flowmetry parameters with accuracy (62.5\%). MF has strong positive correlation with SV $(p=<0.001)$ and

Table 1 Age and BMI of control group and studied cases

\begin{tabular}{lllllll}
\hline Variables & I & II & III & IV & Test of significance & $P$ value \\
\hline Age/years mean $\pm \mathrm{SD}$ & $28.9 \pm 7.23$ & $33.1 \pm 7.17$ & $30.10 \pm 4.51$ & $32.60 \pm 6.11$ & $F=0.99$ & $P=0.41$ \\
BMI $\left(\mathrm{Kg} / \mathrm{m}^{2}\right)$ mean $\pm \mathrm{SD}$ & $32.0 \pm 4.08$ & $32.85 \pm 6.25$ & $30.91 \pm 3.16$ & $27.60 \pm 4.99$ & $F=2.33$ & $P=0.09$
\end{tabular}

BMI body mass index, Sex all are females, SD standard deviation, Groups: I first discovered IIH patients, I/ on medical treatment, III on frequent lumbar tapping, IV control 
Table 2 Parameters of CSF flowmetry between cases and control group

\begin{tabular}{|c|c|c|c|c|c|c|}
\hline \multirow[t]{2}{*}{ CSF flowmetry } & \multirow{2}{*}{$\begin{array}{l}\text { IV } \\
N=10\end{array}$} & \multirow{2}{*}{$\begin{array}{l}I \\
N=10\end{array}$} & \multirow{2}{*}{$\begin{array}{l}\text { II } \\
N=10\end{array}$} & \multirow{2}{*}{$\begin{array}{l}\text { III } \\
N=10\end{array}$} & \multirow[t]{2}{*}{ Test of significance } & \multirow[t]{2}{*}{$P$ value } \\
\hline & & & & & & \\
\hline PSV & $3.0 \pm 0.85$ & $3.47 \pm 1.09$ & $4.40 \pm 2.14$ & $3.30 \pm 1.2$ & $F=1.83$ & $\begin{array}{l}P=0.16 \\
p 1=0.29 \\
p 2=0.07 \\
p 3=0.53\end{array}$ \\
\hline PDV & $2.45 \pm 0.85$ & $2.86 \pm 1.62$ & $3.48 \pm 1.1$ & $2.63 \pm 0.96$ & $F=1.47$ & $\begin{array}{l}P=0.24 \\
\text { p1 }=0.48 \\
\text { p2 }=0.03^{*} \\
\text { p3 }=0.44\end{array}$ \\
\hline SV & $23.30 \pm 7.57$ & $27.05 \pm 13.51$ & $29.65 \pm 9.26$ & $24.35 \pm 12.9$ & $F=0.68$ & $\begin{array}{l}P=0.57 \\
p 1=0.44 \\
\text { p2 }=0.11 \\
\text { p3 }=0.81\end{array}$ \\
\hline Aqueductal area & $0.041 \pm 0.01$ & $0.044 \pm 0.02$ & $0.042 \pm 0.01$ & $0.037 \pm 0.01$ & $F=0.25$ & $\begin{array}{l}P=0.86 \\
\text { p1 }=0.75 \\
\text { p2 }=0.98 \\
\text { p3 }=0.56\end{array}$ \\
\hline MF & $0.11 \pm 0.03^{B}$ & $1.28 \pm 0.04$ & $0.16 \pm 0.047^{A B}$ & $0.096 \pm 0.06^{\mathrm{A}}$ & $F=3.10$ & $\begin{array}{l}P=0.039^{*} \\
\text { p1 }=0.31 \\
\text { p2 }=0.02^{*} \\
\text { p3 }=0.55\end{array}$ \\
\hline
\end{tabular}

All parameters described as mean \pm SD. Similar superscripted letters in same row denote significant difference between groups

*Statistically significant. PSV peak systolic velocity $(\mathrm{cm} / \mathrm{s})$, PDV peak diastolic velocity $(\mathrm{cm} / \mathrm{s})$, SV stroke volume $(\mathrm{ml} / \mathrm{min})$, aqueductal area $\left(\mathrm{cm}{ }^{2}\right), M F \mathrm{mean}$ flow $\left(\mathrm{cm}^{3} / \mathrm{s}\right), p 1$ difference between control and first discovered groups, $p 2$ difference between control and on medical treatment groups, $p 3$ difference between control and on frequent lumber tapping groups

moderate positive correlation with $\operatorname{PSV}(P=0.001)$, $\operatorname{PDV}(P=0.001)$, and aqueductal area $(P=0.005)$. Furthermore, SV and aqueductal area are statistically significant predictors of MF among studied cases $(P<0.05)$ as $67 \%$ of MF can be predicted by SV and area. Yet, after all these finding, opening CSF pressure by lumbar tapping still had the highest sensitivity (92.6\%) and specificity $(100 \%)$ with accuracy reaching $94.6 \%$. The low rate and flow passing through the aqueduct in the patient group may be related to low CSF production following medical treatment.

Bradley and his research group on normal pressure hydrocephalus (NPH) also mentioned that the increased CSF stroke volume is a more important predictor of shunt response than the increased CSF flow void sign, so he found that SV was useful in selecting patients who will actually benefit from shunting as the sensitivity of the aqueductal stroke volume criteria was $80 \%$ and the specificity was $100 \%$ [14]. Moreover, Qvarlander and his colleagues found that mean flow in aqueduct is a more important parameter in CSF dynamic, and this may reflect altered CSF distributions among third and fourth ventricles [15]. But in our study on IIH patients, we found that SV has low sensitivity (56.7\%), specificity (50\%), and accuracy (55\%) than other parameters.

The limitations to our study included that it was a cross-sectional study, and longitudinal study needs to be performed with larger number of patients. Also, followup of CSF dynamics after specific interventions like shunt insertion should be obtained. In this study we

Table 3 Validity of CSF flowmetry and opening pressure in differentiating cases and control

\begin{tabular}{|c|c|c|c|c|c|c|c|c|c|}
\hline \multirow{2}{*}{$\begin{array}{l}\text { Test result } \\
\text { variable(s) }\end{array}$} & \multirow[t]{2}{*}{ AUC } & \multicolumn{2}{|c|}{$95 \%$ confidence interval } & \multirow{2}{*}{$\begin{array}{l}\text { Cutoff } \\
\text { point }\end{array}$} & \multirow[t]{2}{*}{ Sensitivity } & \multirow[t]{2}{*}{ Specificity } & \multirow[t]{2}{*}{ PPV } & \multirow[t]{2}{*}{ NPV } & \multirow[t]{2}{*}{ Accuracy } \\
\hline & & Lower bound & Upper bound & & & & & & \\
\hline PSV & 0.628 & .422 & .833 & $\geq 2.65$ & $83.3 \%$ & $60.0 \%$ & $86.2 \%$ & $54.5 \%$ & $77.5 \%$ \\
\hline PDV & 0.628 & .431 & .825 & $\geq 2.15$ & $76.7 \%$ & $50.0 \%$ & 82.1 & 41.7 & $70.0 \%$ \\
\hline SV & 0.543 & .347 & .738 & $\geq 24.25$ & $56.7 \%$ & $50.0 \%$ & $77.3 \%$ & $27.8 \%$ & $55 \%$ \\
\hline MF & 0.583 & .397 & .769 & $\geq 0.1188$ & $56.7 \%$ & $80.0 \%$ & $69.5 \%$ & $38.1 \%$ & $62.5 \%$ \\
\hline Area & 0.568 & .365 & .772 & $\leq 0.043$ & $70.0 \%$ & $50.0 \%$ & $80.8 \%$ & $35.7 \%$ & $65 \%$ \\
\hline Opening pressure & 1.000 & 1.000 & 1.000 & $\geq 26.0$ & $92.6 \%$ & $100.0 \%$ & $100 \%$ & $83.3 \%$ & $94.6 \%$ \\
\hline
\end{tabular}

AUC area under the curve, NPV negative predictive value, PPV positive predictive value, PSV peak systolic velocity (cm/s), $P D V$ peak diastolic velocity (cm/s), SV stroke volume $(\mathrm{ml} / \mathrm{min})$, aqueductal area $\left(\mathrm{cm}^{2}\right), M F$ mean flow $\left(\mathrm{cm}^{3} / \mathrm{s}\right)$ 
Table 4 Linear regression for prediction of MF among studied cases

\begin{tabular}{|c|c|c|c|c|c|c|}
\hline \multirow[t]{2}{*}{ Model } & & \multicolumn{2}{|c|}{ Unstandardized coefficients } & \multirow{2}{*}{$\begin{array}{l}\text { Standardized coefficients } \\
\text { Beta }\end{array}$} & \multirow[t]{2}{*}{$t$} & \multirow[t]{2}{*}{$P$} \\
\hline & & $B$ & Standard error & & & \\
\hline \multirow[t]{6}{*}{1} & (Constant) & -.035 & .029 & & -1.207 & 0.239 \\
\hline & PSV & .010 & .005 & .294 & 1.962 & 0.061 \\
\hline & PDV & .007 & .007 & .167 & 1.041 & 0.308 \\
\hline & SV & .002 & .001 & .437 & 2.672 & 0.013 \\
\hline & Area & 1.162 & .449 & .380 & 2.591 & 0.016 \\
\hline & $\begin{array}{l}F=12.9, P<0.001^{*} \\
R^{2}=0.67\end{array}$ & & & & & \\
\hline
\end{tabular}

PSV peak systolic velocity $(\mathrm{cm} / \mathrm{s}), P D V$ peak diastolic velocity $(\mathrm{cm} / \mathrm{s}), S V$ stroke volume $(\mathrm{ml} / \mathrm{min})$, aqueductal area $\left(\mathrm{cm}^{2}\right)$

concentrate on monitor CSF flow dynamics changes with attempt to consider it as dependable noninvasive diagnostic tool for IIH patients.

\section{Conclusion}

PCC-MRI is a non-invasive method used to study CSF flow dynamics in IIH. The most specific CSF flowmetry parameter to diagnose $\mathrm{IIH}$ is mean flow (MF), and it was significantly higher in first discovered IIH group than other IIH groups. So we concluded that PCC-MRI can be used as non-invasive technique for diagnosis of IIH. Also, the PCC-MRI may be used as noninvasive tool to follow-up the patient response to treatment. A further study on large scale of patients with different demographic parameters should be done.

\section{Abbreviations}

IIH: Idiopathic intracranial hypertension; MF: Mean flow; PCC-MRI: Phase contrast cine MRI; NPH: Normal pressure hydrocephalus; CSF: Cerebrospinal fluid; BMI: Body mass index; SD: Standard deviation; PSV: Peak systolic velocity; PDV: Peak diastolic velocity

\section{Acknowledgements}

The authors acknowledge subjects for their participation and cooperation in this study.

\section{Authors' contributions}

A.R. contributed in collecting data and diagnosing cases and writing the manuscript. T.B. regularly revised the sample collection, diagnosis, analyzed the results and prepared the manuscript in its full presentation. A.A. supervised the final work and revised the study outcomes. F.S. author was involved in MRI studies and diagnosing radiological images. All authors have read and approved the final manuscript.

\section{Funding}

There is no source of funding for the research. This study was this research was supported by the Department of Neurology, Faculty of Medicine, at Mansoura University as regard patient admission if they were in need for that

\section{Availability of data and materials}

The datasets generated and or analyzed during the current study are not publicly available due to current Mansoura University regulations and Egyptian legislation but are available from the corresponding author on reasonable request and after institutional approval.

\section{Competing interest}

All authors declare that they have no conflict of interest.
Ethics approval and consent to participate

All procedures performed in studies involving human participants were in accordance with the ethical standards of the institutional research committee and with the 1964 Helsinki declaration and its later amendments or comparable ethical standards. The study was accepted by IRB, Faculty of Medicine, Mansoura University, under the code number of MS / 17.07.13 on Aug. 8, 2017.

An informed written consent was taken from each participant involved in this study prior to the conduct of any study-related activities.

All data obtained from participants were confidential and were not used outside the study. The patients had the rights to withdraw from the study at any time without giving any reason.

We agree to publish in the EJNPN, and the research is only applied here.

Consent for publication

Not applicable

\section{Author details}

${ }^{1}$ Department of Neurology, Faculty of Medicine, Mansoura University, Mansoura 35516, Egypt. ${ }^{2}$ Department of Diagnostic Radiology, Mansoura University, Mansoura, Egypt.

Received: 19 December 2019 Accepted: 9 September 2020 Published online: 18 September 2020

\section{References}

1. Kosmorsky GS. Idiopathic intracranial hypertension: pseudotumor cerebri. Headache. 2014;54(2):389-93.

2. Mollan SP, Ali F, Hassan-Smith G, Botfield H, Friedman DI, Sinclair AJ. Evolving evidence in adult idiopathic intracranial hypertension: pathophysiology and management. J Neurol Neurosurg Psychiatry. 2016; 87(9):982-92.

3. St MI, lencean AS, Tascu A. Pseudotumour cerebri idiopathic intracranial hypertension and vascular intracranial hypertension. Rom J Neurosurg. 2015; 29(4):397-409.

4. Rogers DL. A review of pediatric idiopathic intracranial hypertension. Pediatr Clin N Am. 2014;61(3):579-90.

5. Fin $L$, Grebe R. Three dimensional modeling of the cerebrospinal fluid dynamics and brain interactions in the aqueduct of Sylvius. Comput Methods Biomech Biomed Engin. 2003;6(3):163-70.

6. Chikly B, Quaghebeur J. Reassessing cerebrospinal fluid (CSF) hydrodynamics: a literature review presenting a novel hypothesis for CSF physiology. J Bodyw Mov Ther. 2013;17(3):344-54.

7. Wall M. Idiopathic intracranial hypertension. Neurol Clin. 2010;28(3):593-617.

8. Smith J. Whence pseudotumor cerebri? J Clin Neuro Ophthalmol. 1985;5(1):55.

9. Lindstrøm EK, Ringstad G, Mardal K-A, Eide PK. Cerebrospinal fluid volumetric net flow rate and direction in idiopathic normal pressure hydrocephalus. Neuroimage Clin. 2018;20:731-41.

10. Bulat M, Klarica M. Recent insights into a new hydrodynamics of the cerebrospinal fluid. Brain Res Rev. 2011;65(2):99-112.

11. Corp I. IBM SPSS statistics for windows, version 22.0. IBM Corp.: Armonk, NY. 2013.

12. Akay R, Kamisli O, Kahraman A, Oner S, Tecellioglu M. Evaluation of aqueductal CSF flow dynamics with phase contrast cine MR imaging in 
idiopathic intracranial hypertension patients: preliminary results. Eur Rev Med Pharmacol Sci. 2015;19(18):3475-9.

13. Cappuzzo JM, Hess RM, Morrison JF, Davies JM, Snyder KV, Levy El, et al. Transverse venous stenting for the treatment of idiopathic intracranial hypertension, or pseudotumor cerebri. Neurosurg Focus. 2018;45(1):E11.

14. Bradley W. Idiopathic normal pressure hydrocephalus: new findings and thoughts on etiology. AJNR Am J Neuroradiol. 2008;29(1):1.

15. Qvarlander S, Ambarki K, Wåhlin A, Jacobsson J, Birgander R, Malm J, et al. Cerebrospinal fluid and blood flow patterns in idiopathic normal pressure hydrocephalus. Acta Neurol Scand. 2017;135(5):576-84.

\section{Publisher's Note}

Springer Nature remains neutral with regard to jurisdictional claims in published maps and institutional affiliations.

\section{Submit your manuscript to a SpringerOpen ${ }^{\circ}$ journal and benefit from:}

- Convenient online submission

- Rigorous peer review

- Open access: articles freely available online

High visibility within the field

- Retaining the copyright to your article

Submit your next manuscript at $\boldsymbol{\nabla}$ springeropen.com 